\title{
LA-UR-01-0509
}

Los Alamos National Laboratory is operated by the University of California for the United States Department of Energy under contract W-7405-ENG-36.

\section{TITLE: $\quad$ Multiscale Modeling of Air Flow in Salt Lake City and the Surrounding Region}

\author{
AUTHOR(S): Michael Brown, LANL \\ Marty Leach, LLNL \\ Ron Calhoun, LLNL \\ Scott Smith, LANL \\ David Stevens, LLNL \\ Jon Reisner, LANL \\ Bob Lee, LLNL \\ Steve Chin, LLNL \\ David DeCroix, LANL
}

SUBMITTED to: ASCE Structures Congress 2001, Washington, DC May, 2001 


\title{
Multiscale Modeling of Air Flow in Salt Lake City and the Surrounding Region
}

\author{
Michael Brown ${ }^{1 *}$, Marty Leach ${ }^{2}$, Ron Calhoun ${ }^{2}$, Scott Smith $^{1}$, David Stevens ${ }^{2}$, \\ Jon Reisner ${ }^{1}$, Bob Lee ${ }^{2}$, Steve $_{\text {Chin }}^{2}$, \& David DeCroix ${ }^{1}$ \\ ${ }^{1}$ Los Alamos National Laboratory, Los Alamos, New Mexico \\ ${ }^{2}$ Lawrence Livermore National Laboratory, Livermore, California \\ * LANL, Group TSA-4, MS F604, Los Alamos, NM 87545; 505-667-1788, mbrown@lanl.gov
}

\begin{abstract}
A general overview is given of a modeling effort to simulate the fate and transport of a tracer within the downtown core of Salt Lake City and beyond into the Salt Lake Basin. The problem crosses three significant scales where different physics are predominant: atmospheric mesoscale, city scale, and building scale. Three different computational fluid dynamics models were used, each with strengths at particular spatial and temporal scales. We show preliminary results and discuss what we believe to be the relevant phenomenon one must model as one crosses from atmospheric scale to engineering scale flow problems. We also describe our model validation efforts, including wind-tunnel and tow-tank experiments and a recently completed urban field experiment.
\end{abstract}

\section{Introduction}

For plumes originating in urban areas, the larger-scale transport may depend on the near-source dispersal patterns within the city. In addition, the near-source dispersal behavior within the urban canopy may depend on the larger-scale flow features. There have been a large number of studies on simulations of flows around buildings (e.g., Murakami, 1993; Selvam, 1996; Calhoun et al., 2000a) and on the mesoscale that have included urban canopy effects (see review by Brown, 2000). Fewer simulations have been performed linking building-scale and mesoscale models (e.g., Brown and Müller, 1997; Cox et al., 2000), and even fewer have been conducted that explicitly simulate the flow on the individual building scale, the many-building urban scale, and the mesoscale. Below we describe a collaborative effort between Lawrence Livermore and Los Alamos National Laboratories in which a system of models are linked through boundary conditions in order to study a problem from the large mesoscale down to the building scale. In this paper, we will present preliminary meteorological and tracer dispersion simulations from a hierarchy of three prognostic models that were centered around Salt Lake City, Utah. We cover the mesoscale, the urban scale, and the building scale using the models in a nested configuration. The multi-scale modeling approach allows us to explicitly capture mesoscale flow features over the large domain as well the effects of individual buildings in the smaller area of interest. We also present simulation results from high resolution basin scale and urban scale simulations using climatological winds in order to better understand the flow dynamics during a field experiment that was performed in Salt Lake City in October, 2000. 


\section{Model Descriptions}

a) COAMPS. The Naval Research Laboratory's 3-D “Coupled Ocean/Atmosphere Mesoscale Prediction System" solves the geophysical fluid equations for atmospheric momentum, heat transport, moisture, and surface energy budget. COAMPS consists of a data assimilation system, a nonhydrostatic atmospheric forecast model, and a hydrostatic ocean model. In this study, we use only the atmospheric model, which solves the compressible form of the dynamical equations and has a nested-grid capability and parameterizations for subgrid-scale mixing, surface momentum and heat fluxes, explicit ice microphysics, subgrid-scale cumulus clouds, and shortwave and longwave radiation. A terrain-following vertical coordinate is used to simulate flow over an irregular surface. An urban canopy parameterization is incorporated (Chin et al, 2000). The reader is referred to Hodur (1997) for further details on COAMPS.

b) HIGRAD. The "High Gradient" model solves the 2-D Navier-Stokes equations in a terrain-following coordinate system. The model is second-order accurate and uses a non-oscillatory forward-in-time advection scheme that can accurately model regions of strong shear. The model can be run in an anelastic mode using an efficient conjugate residual pressure solver or in a compressible mode using the method of averages. Turbulence closure is accomplished using a Smagorinsky-type or a TKE-based large eddy simulation (LES) scheme. The code solves a surface energy budget equation and includes shading effects. Further information can be found in Reisner et al. (1998).

c) FEM3MP. The "Finite Element Model 3 - Massively Parallel" solves the 3-D Navier-Stokes equations. An anelastic approximation allows the model to simulate a wide range of stability conditions. An implicit time discretization scheme means that larger timesteps can be taken. The model incorporates an advanced multigrid Poisson solver. There are two principal turbulence models: a LES Smagorinsky turbulence model with a special treatment of the subgrid length scale in the presence of buildings and a three equation RANS model which contains many of the features of second-order closure. A more thorough description of the FEM3MP model can be found in Gresho and Chan (1998).

\section{Experimental Descriptions}

Validation and testing of the models are a key component to our study program. We have conducted wind-tunnel and field experiments to help with this task, in addition to utilizing data from earlier studies. We briefly describe the experiments below.

a) Wind-tunnel Experiments. Wind-tunnel experiments of 2-D and 3-D arrays of buildings in a deep "atmospheric" boundary layer were performed in the USEPA meteorological wind tunnel (Lawson et al., 2000). Inflow is turbulent and the velocity profile can be described by a power law. Relatively high resolution measurements of the mean and turbulent velocity components were measured upstream, downstream, within and above the building arrays using a pulsed wire anemometer. Pressure measurements were obtained on the upwind, rooftop, and downwind face of each building in the 2-D array. Concentration measurements were taken downwind of a point source release within the 3-D building array. The velocity measurements around the 2-D array are described in Brown et al. (2000). 
b) Tow-tank Experiment. Experiments of tracer dispersion around a single cubical obstacle were performed in the USEPA stratified towing tank. A salt solution is used to stratify the water and the model (in this case, a cube) is towed the length of the tow tank channel upside down along the water surface. "Flow" past the block is stratified and laminar, and the velocity profile is constant with height. For several different stably stratified conditions, concentration measurements were collected downstream of the cube for point source releases behind the cube. Cube cavity length was also measured as a function of Froude number. Further information on the experiment can be found in Snyder (1994).

c) Field Experiment. A major field campaign called URBAN1 was held in Salt Lake City, Utah during the month of October, 2000 (Shin et al., 2000). Tracer and meteorological data were collected during the night over three different transport scales: building, city, and regional scales. The field experiment was closely coordinated with the DOE's regional-scale Vertical Transport and Mixing (VTMX) experiment performed in the greater Salt Lake City area. Six tracer experiments were conducted using perfluorocarbon (PFTs) and sulfur hexafluoride (SF6) tracers. Timeintegrated tracer samples (nominally 5-minute to 2-hour integration times) were collected by 200 samplers located throughout the Salt Lake Basin (45 SF6 samplers were located around the downtown study building, 40 SF6/PFT samplers and 24 SF6 samplers were located in a 5-block-square area (25 blocks) of downtown, 36 SF6 samplers were located on three sampling arcs (2-, 4-, and 6-km) to the northwest of the downtown SF6 release location, and 55 PFT samplers were located throughout the Salt Lake Basin). In addition, two fast-response SF6 analyzers were located around the downtown study building, and six mobile fast-response SF6 analyzers were operated within the downtown study area and slightly beyond. Meteorological measurements made during the URBAN1/VTMX field experiment included surface weather at 29 sites (plus 18 existing sites), vertical profiles of wind and temperature (8 radar profilers, 10 acoustic sodars, 4 rawinsonde balloon systems, 4 tethered-balloon systems), temperature measurements at one block intervals across the city along two lines (54 temperature data loggers plus one mobile unit), velocity and turbulence measurements around select buildings using 33 sonic anemometers, and wind fields aloft obtained with two doppler lidars.

\section{Model Setup}

a) Setup1 - Linked Models. The COAMPS model utilized a 36, 12, and $4 \mathrm{~km}$ resolution nested grid mesh and was run for a 36 hour forecast with a begin time of 5:00 am Dec. 9, 1999. The outermost mesh covered the western US, while the innermost mesh covered a 240x240 km area in the Salt Lake City basin. Wind, temperature, and humidity profiles computed by COAMPS were used to drive the HIGRAD urban-scale simulation. Results presented in this paper represent a simulation covering a 1.6x1.5 km domain in downtown Salt Lake City with $10 \mathrm{~m}$ horizontal grid size and variable spacing in the vertical (minimum of $3 \mathrm{~m}$ at the surface). HIGRAD-produced meteorological profiles were then used to drive the FEM3MP model at grid resolutions on the order of meters. Here, the flow field around individual buildings was resolved at high resolution. Below we show examples of these simulations at each scale.

b) Setup2 - Basin scale. In order to learn more about the physics and resolution requirements for basin-scale flows, we performed some preliminary basin-scale calculations using typical wind speeds and directions for the Salt Lake basin using both the FEM3MP and HIGRAD models. In the calculations we are going to present in this paper using the FEM3MP code, the surface cool- 
ing is set to a fixed $-20 \mathrm{~W} / \mathrm{m}^{2}$ and the inflow is logarithmic and neutrally stratified. For computational efficiency, the overall calculation was composed of three parts: a coarse ramp-up calculation utilizing 7 million grid points, a fine resolution run using 50 million grid points, and a very fine resolution run of 400 million grid points. Each higher resolution run was an interpolated, continuation of the previous coarser calculation. Horizontal grid resolution was $100 \mathrm{~m}, 50$ $\mathrm{m}$, and $25 \mathrm{~m}$, respectively, for the coarse, fine, and very fine resolution runs. Vertical grids were stretched with finest resolution at the surface of $4 \mathrm{~m}, 2 \mathrm{~m}$, and $1 \mathrm{~m}$, respectively. The domain size was approximately 50 kilometers on a side and extended $2.8 \mathrm{~km}$ above the surface. The coarse scale simulation began slightly before sunset and ran for 6 hours into the night. The fine scale simulation ran for about 2 hours interpolating from hour number 3 from the coarse scale simulation. To date, the super fine scale simulation has only been run for about 2 minutes. The three simulations were run using 20,40, and 100 nodes on the ASCI White massively parallel computing platform located at Lawrence Livermore National Laboratory, where each node typically consisted of 10 to 14 processors.

c) Setup3 - Urban scale. Pre-field experiment simulations of the transport and dispersion of several tracer releases in the downtown Salt Lake City area have been conducted using the HIGRAD and FEM3MP models in order to examine the effect of groups of buildings on the overall tracer transport in downtown Salt Lake City. The run shown in this paper was conducted on a $216 \times 192$ x 51 grid mesh. The grid resolution was $10 \mathrm{~m}$ in the horizontal and varied in the vertical, with the finest resolution being $2.5 \mathrm{~m}$ near the surface. The top of the model domain was $1 \mathrm{~km}$. A uniform potential temperature profile was used at the inflow boundary, and several runs were performed with uniform southeasterly winds with speeds ranging between 0.5 to $3 \mathrm{~m} / \mathrm{s}$. Simulations were performed using 64 processors on LANL's ASCI Blue Mountain massively parallel computing platform.

d) Setup 4 - Wind tunnel. Both the HIGRAD and FEM3MP models are being tested against wind tunnel and tow tank data (Smith et al., 2001; Chan et al., 2000). For illustrative purposes, we show a few comparisons of HIGRAD model computations using large eddy simulation (LES) and wind-tunnel measurements of a "2-D" array of wide buildings. The LES simulations were conducted on a domain of 360 x 128 x 63 grids (longitudinal, lateral, and vertical directions, respectively). The grids were evenly spaced horizontally with the building rows being resolved at 0.1 $\mathrm{Hb}$ (building height) in the longitudinal and $0.2 \mathrm{Hb}$ in the lateral. The vertical grid spacing varied with height with the highest resolution near the surface. Buildings were resolved vertically with 23 grids. A laminar, neutrally-stratified wind profile determined from the wind-tunnel data was used as the inflow boundary condition. A simple law-of-wall parameterization for the momentum flux was used at the wind-tunnel floor and building surfaces. At least 4 seconds of simulation were performed to allow for sufficient model spinup, and modeled mean and turbulent flow characteristics were averaged over a period of at least 20 seconds. Simulations were performed using 80 processors on the ASCI Blue Mountain computing platform.

e) Setup5 - Tow tank. For the tow tank experiments using the HIGRAD model, a $60 \mathrm{~m}$ cubical building was modeled in a 3-D domain covering $750 \mathrm{~m}$ x $350 \mathrm{~m}$ x $250 \mathrm{~m}$. Grid size was constant at $5 \mathrm{~m}$. A uniform laminar inflow wind profile of $7 \mathrm{~m} / \mathrm{s}$ was imposed. The simulations were for one hour using time steps of $0.5 \mathrm{~s}$ or less. Simulations were performed on the ASCI Blue Mountain computing platform. 


\section{Discussion}

a) Linked modeling. Figure 1 shows the COAMPS-computed wind field on the innermost mesh at $10 \mathrm{~m}$ above ground level (agl) for $5 \mathrm{am}$, Dec. 10, 1999. Drainage flow has developed due to the mountains and stagnation occurs over the city. Since the mesoscale grid is too large to resolve buildings, we have implemented urban canopy parameterizations into the COAMPS code that account for the drag and turbulence production associated with buildings, as well as radiation and surface energy budget effects (Chin et al., 2000). With the urban canopy parameterization turned off, the urban area does not impede the drainage flow and the stagnation zone is less evident. Vertical profiles of velocity, temperature, and turbulent kinetic energy (TKE) over the city are altered significantly by the urban canopy. Figure 2 shows an example of the effect of the urban canopy parameterizations on circulation and plume dispersion. Qualitatively, the circulation is similar in the simulation with or without the urban effects included. However, the strength of the circulation is affected by the presence of the urban area. Southeasterly flow through the middle of the domain and the drainage flow off of the mountains are much weaker when the urban effects are present. The resulting effect on plume transport and dispersion is evident in the picture. A simulated tracer was released at the point marked $\mathbf{X}$ for 30 minutes beginning at 5 AM local time; the results are shown for $8 \mathrm{AM}$ local time. Without the urban effects, the plume travels farther both away from the source (stronger drainage flow) and towards the northwest (in the southeasterly flow).

Vertical profiles of spatially-averaged COAMPS wind, temperature, moisture and TKE over one grid cell in the Salt Lake City area for 5 am were used as the boundary conditions for the HIGRAD code. Here we assumed that the meteorological fields did not change appreciably over the one hour period of HIGRAD simulation. Figure 3 shows the wind fields at $10 \mathrm{~m}$ agl over the southeastern quadrant of the domain for 6 am. Clearly, very complicated wind patterns have developed among the irregularly arranged group of buildings. The HIGRAD wind and turbulence fields were area-and time averaged and vertical profiles provided to FEM3MP. Figure 4 shows a steady-state solution for the wind field around the Delta Center in Salt Lake City. A double vortex forms on the downstream side which is slightly skewed due to the presence of the two small buildings. Simulations of tracer dispersion show significant lofting of the plume due to the buildings. Our preliminary results illustrate the effects of including urban areas on the locally forced, mesoscale wind fields, and at finer scales of including individual buildings and building clusters. As we continue this work, we will investigate the necessity of having time-dependent and spatially-varying boundary effects. Currently our efforts are focused on simulating several case days during the URBAN field experiment.

b) Urban scale simulations. In order to help with sensor placement, simulations were performed prior to the field experiment in the Salt Lake City downtown area with both the FEM3MP and HIGRAD codes. Using local wind climatology, simulations were performed with prevailing wind conditions and for several tracer release points. In the example shown in Fig. 5, a light southeasterly wind is advecting the tracer to the northwest. However, transport is also occurring at oblique angles to the prevailing wind due to significant channeling within the street canyons. 3-D visualization shows that significant lofting of the tracer occurs in the wakes of the tall buildings as well. These simulations used fixed boundary conditions. Future simulations will incorporate time and spatially varying boundary conditions obtained from basin scale simulations (see next sub-section). 
There are several interesting issues that arise at the urban scale. One is that the urban scale is in between the traditional smaller engineering scales and the larger atmospheric scales. Phenomena familiar to meteorologists such as the Coriolis force, stratification, and surface energy budget are still important at the urban scale. For example, Fig. 6 shows the effect of the surface energy budget, that is the natural heating (and cooling) of the earth's surface due to short and longwave radiation, on transport and dispersion of a tracer for a Washington DC simulation. Small scale effects such as roof pitch, differential heating of wall and road surfaces, and porosity of building structures may also be important. For example, simulations by Smith et al. (2000) have shown that solar heating of a building's walls changes the character of flow and dispersion around the building. Another interesting issue at the urban scale is the effect of grid size. At the urban scale we cannot computationally afford to have high resolution everywhere, but we are aware that coarse grid resolution may not resolve the significant features of the flow. Large-eddy simulation is especially sensitive to grid size, as shown by single building simulations by DeCroix et al. (2000) (Fig. 7). We are currently investigating the sensitivity of simulations of clusters of buildings to grid size and turbulence closure method.

c) Basin scale simulations. Based on preliminary analyses of the URBAN field experiment data, there appears to be significant variations in the wind direction within Salt Lake City over hourly time scales during the night. It has been hypothesized that this might be due to larger basin scale "sloshing" of the flow and/or due to different drainage currents becoming prominent at different times during the night. In order to better understand the flow characteristics within the city, the basin scale meteorology must be better understood. We have performed some preliminary basinscale calculations with both HIGRAD and FEM3MP, and present FEM3MP results here. Using the prevailing nighttime wind conditions (south to north) and a specified surface cooling of -20 $\mathrm{W} / \mathrm{m}^{2}$, we have performed simulations for about 6 hours after sunset. As shown in Fig. 8, the flow through the downtown area (for typical winter-time conditions) is a complex combination of nocturnal drainage currents which may run counter to the larger-scale flow moving up the valley to the north. These early results suggest that in order to understand the movement of air, and, consequently dispersion, in the downtown Salt Lake city area, sufficient resolution is required in the neighboring valleys to capture the drainage currents which may penetrate into the city at night. As outlined in Calhoun et al. (2000b), further studies are proposed to address LES resolution issues in stable conditions.

d) Wind tunnel validation. Extensive model testing with wind tunnel data is ongoing. In Fig. 9, model-computed wind vectors around the 2-D building array are compared to measurements and show reasonable agreement. In this example, a Smagorinsky-type LES subgrid closure was used. The effect of this subgrid closure was assessed by varying the length scale (or, alternately, the Smagorinsky constant) and it's value proved to be crucial in reproducing the correct mixing and recirculation patterns at roof top and in the street canyons. These recirculation patterns were sensitive to the characteristics of the jet that forms due to separation at the upwind corner of the first building and it's entrainment of the air above the first building rooftop.

e) Tow tank validation. Both the HIGRAD and FEM3MP models have been compared to the stably stratified tow tank experiments of Snyder (1994). Figure 10 shows the reattachment length behind the cubical building as a function of the Froude no. measured in the tow tank experiment and computed by the HIGRAD model. A lateral profile of concentration measurements down- 
stream of the building shows reasonable agreement between model and measurements (Fig. 11). Using the FEM3MP model, Chan et al. (2000) showed that the spread of the tracer behind the building was significantly underestimated using the traditional RANS k- $\varepsilon$ turbulence closure model, whereas large eddy simulation yielded better results.

\section{Conclusions}

We have presented a demonstration of linking model simulations across the mesoscale, urban scale, and building scale. Our preliminary results illustrate the effects of including urban areas on the locally forced, mesoscale wind fields, and at finer scales of including individual buildings and building clusters. As we continue this work, we will include time-dependent and spatially-varying boundary effects. Model validation and testing is ongoing, using wind-tunnel, tow-tank, and field experiment data. A number of significant issues are being addressed at the urban scale, including engineering vs. meteorological CFD, turbulence closure methods, boundary conditions and grid size dependence.

\section{References}

Brown, M. (2000) Urban parameterizations for mesoscale models, from Mesoscale Atmospheric Dispersion, Boybeyi, ed., Wessex Press.

Brown, M. and C. Müller (1997) The effect of microscale urban canyon flow on mesoscale puff dispersion, 12th AMS Symp. Bound. Layers \& Turb., Vancouver, BC.

Brown, M., R. Lawson, D. DeCroix, and R. Lee (2000) Mean flow and turbulence measurements around a 2-D array of buildings in a wind tunnel, 11th AMS Conf. Air Poll. Meteor., Long Beach, CA.

Calhoun, R., S. Chan, R. Lee, J. Leone, J. Shinn, and D. Stevens (2000a) Flow patterns around a complex building, 11th AMS Conf. Appl. Air Poll. Meteor., Long Beach, CA.

Calhoun, R., R. Cederwall, D. Stevens, and R. Street (2000b) Highly-resolved LES of the stable boundary layer over terrain, 14th AMS Symp. Bound. Layers \& Turb., Boulder, CO.

Chan, S. T., R. L. Lee and D. S. Stevens (2000) A Model for Flow and Dispersion Around Buildings and its Validation Using Laboratory Experiments, 3rd AMS Urban Env. Symp., Davis, CA, LLNL Report JC-137458.

Chin S., M. Leach, and M. Brown (2000) A sensitivity study of the urban effect on a regionalscale model: an idealized case, 3rd AMS Urb. Env. Symp., Davis, CA.

Cox, C., B. Cybyk, J. Boris, Y. Fung, and S. Chang (2000) Coupled microscale-mesoscale modeling of contaminant transport in urban environments, 3rd AMS Urb. Env. Symp., Davis, CA.

DeCroix, D., W.S. Smith, G. Streit, and M. Brown (2000) Large-eddy and Gaussian simulations of downwind dispersion from large building HVAC exhaust, 11th AMS Conf. Air Poll. Meteor., Long Beach, CA.

Gresho, P. and S. Chan (1998) Projection 2 goes turbulent and fully implicit. Int. J. Comp. Fluid Dyn. 9, 249-272.

Hodur, R. (1997) The Naval Research Laboratory's coupled ocean-atmospheric mesoscale prediction system (COAMPS). Mon. Wea. Rev. 125, 1414-1430.

Lawson, R., S. Perry, and R. Thompson (2000) Measurement of velocity and concentration fields in arrays of 2-dimensional and 3-dimensional buildings in a simulated neutrally-buoyant atmospheric boundary layer, USEPA FMF data report.

Murakami, S. (1993) Comparison of various turbulence models applied to a bluff body, J. Wind 
Eng. Ind. Aerodyn. 46\&47, 21-36.

Reisner, J., W. Smith, J. Bossert, and J. Winterkamp (1998) Tracer modeling in an urban environment, 2nd AMS Urb. Env. Symp., Albuquerque, NM.

Selvam, P. (1996) Computation of flow around Texas Tech building using k- $\omega$ and Kato-Launder k- $\varepsilon$ turbulence model, Eng. Struct., 18, 856-860.

Shinn, J., K. Allwine, and G. Streit. (2000) The DOE URBAN Field Program for 2000-2004, 3rd AMS Urb. Env. Symp., Davis, CA.

Smith, W.S., J. Reisner, and J. Kao (2001) Simulations of flow around a cubical building: comparison with towing tank data and assessment of radiatively-induced thermal effects, LA-UR-005208, submitted to Atmospheric Environment.

Smith, W.S., J. Reisner, D. DeCroix, M. Brown, R. Lee, S. Chan, and D. Stevens (2000) A CFD intercomparison and validation using high resolution wind tunnel data, 11th AMS Conf. Air Poll. Meteor., Long Beach, CA.

Snyder, W.H. (1994) Some observations of the influence of stratification on diffusion in building wakes, Stably Stratified Flows: Flow and Dispersion of Topography, I.P. Castro and N.J. Rockliff (Eds.), 325-358. 


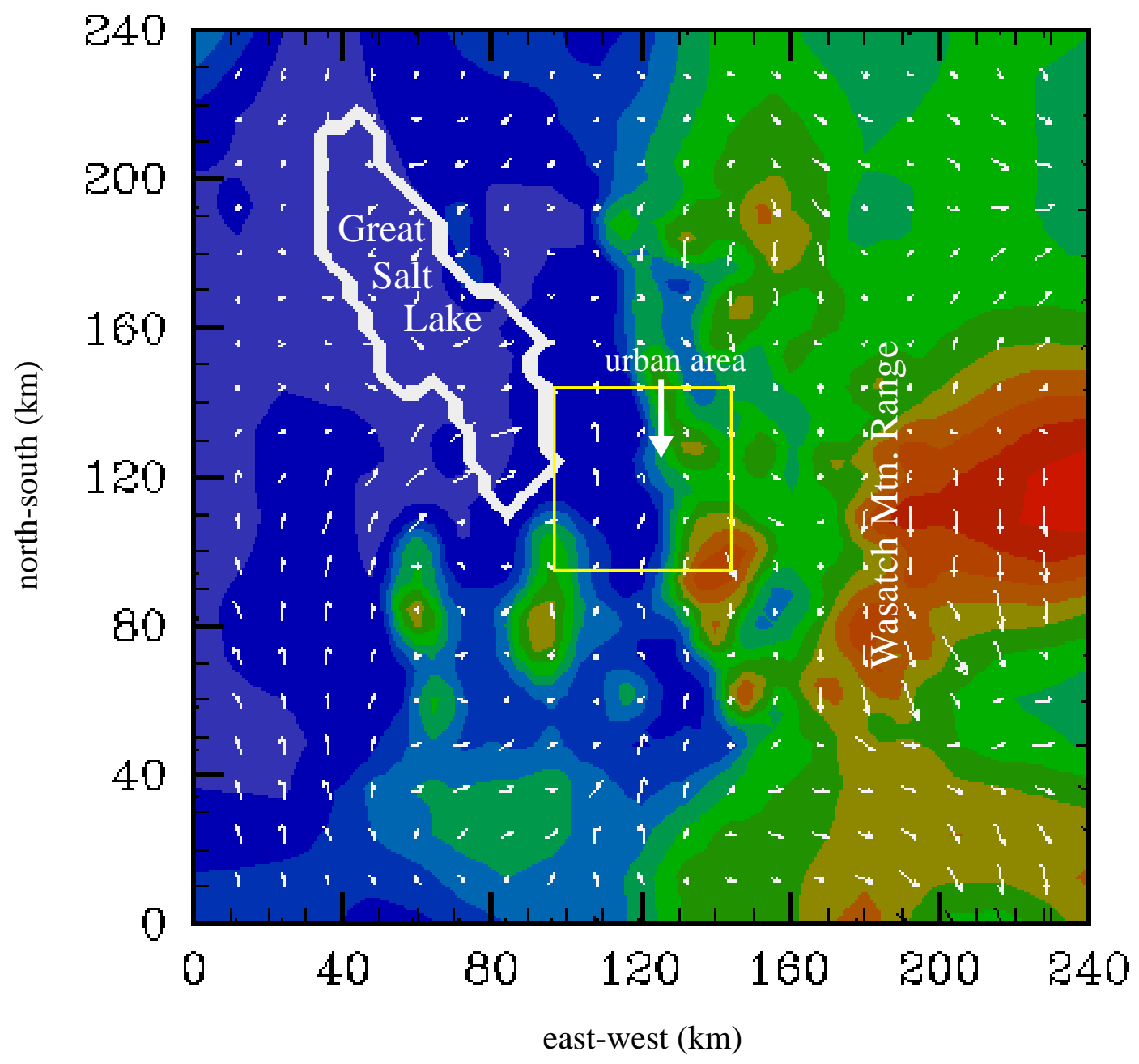

Figure 1. COAMPS regional scale simulation showing early morning near-surface wind patterns in the Salt Lake City basin. Yellow box demarcates domain in Fig. 2.
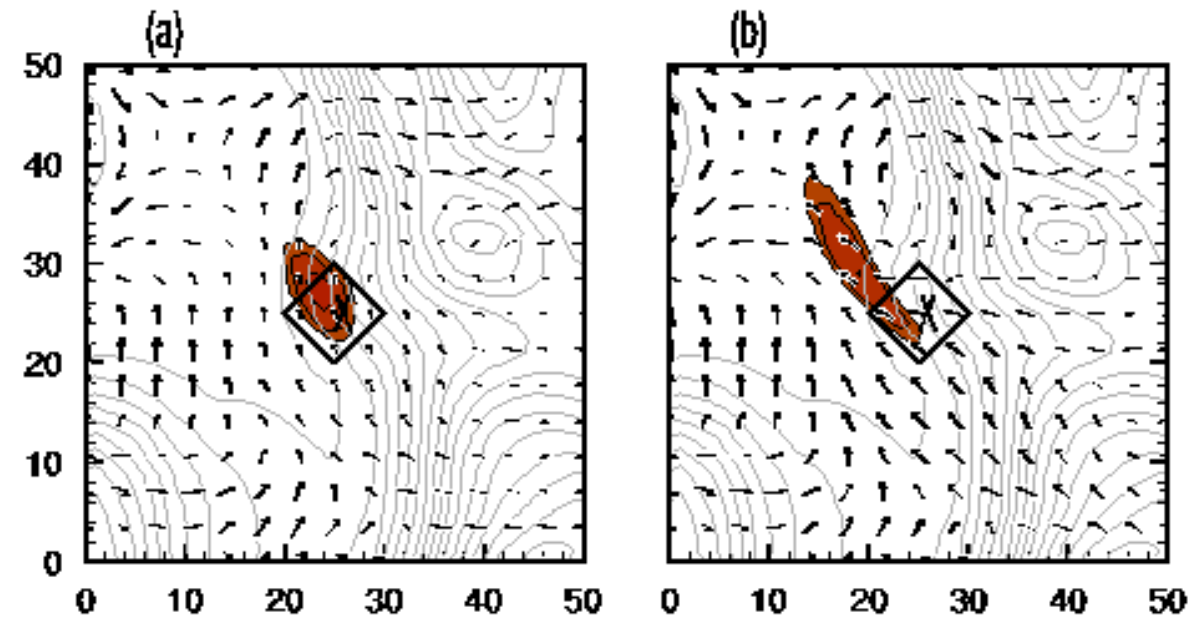

Figure 2. COAMPS/LODI simulation depicting plume transport in the Salt Lake City basin a) with and b) without urban canopy parameterizations implemented in the numerical model. 


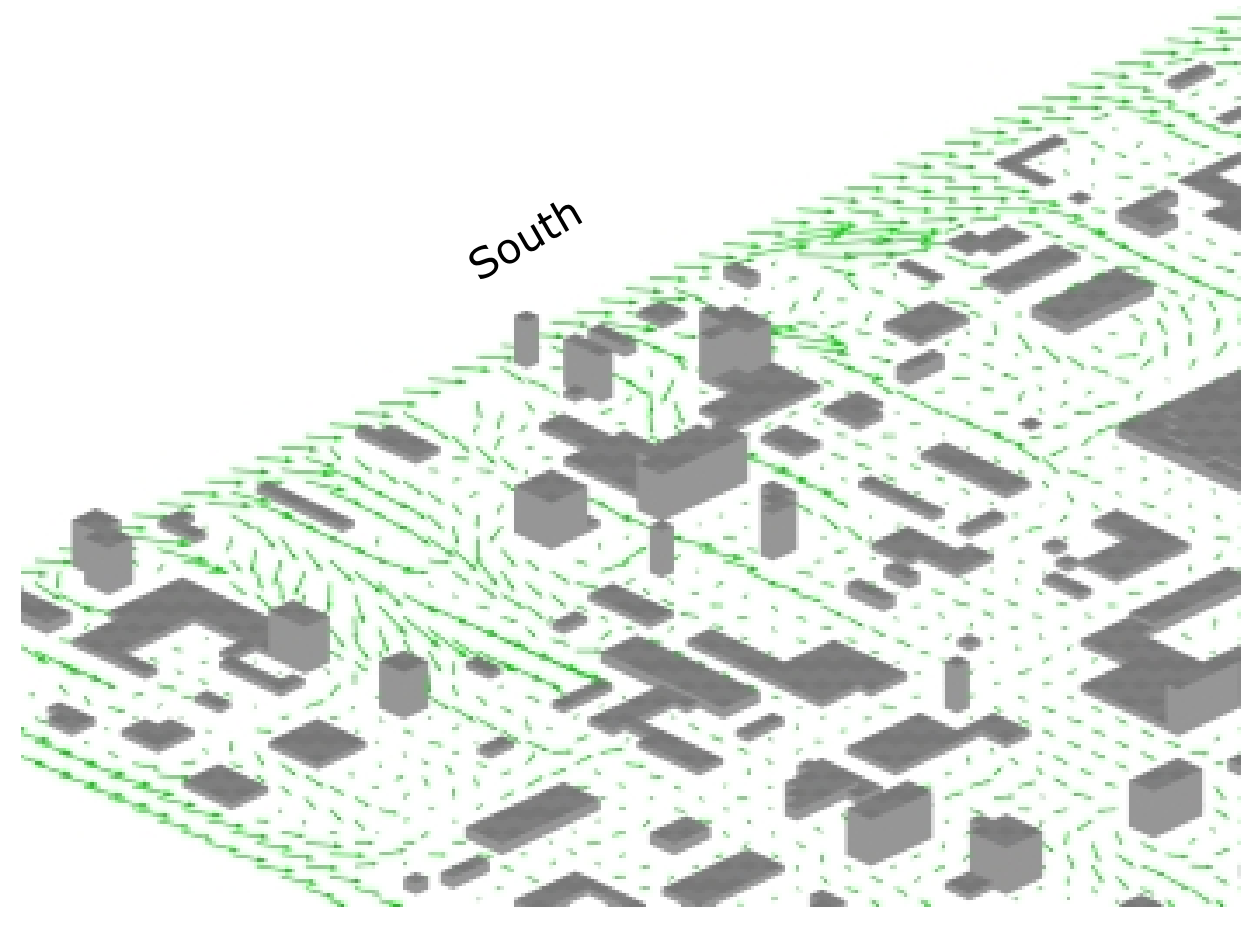

Figure 3. HIGRAD urban scale simulation showing wind vectors around buildings in downtown Salt Lake City.

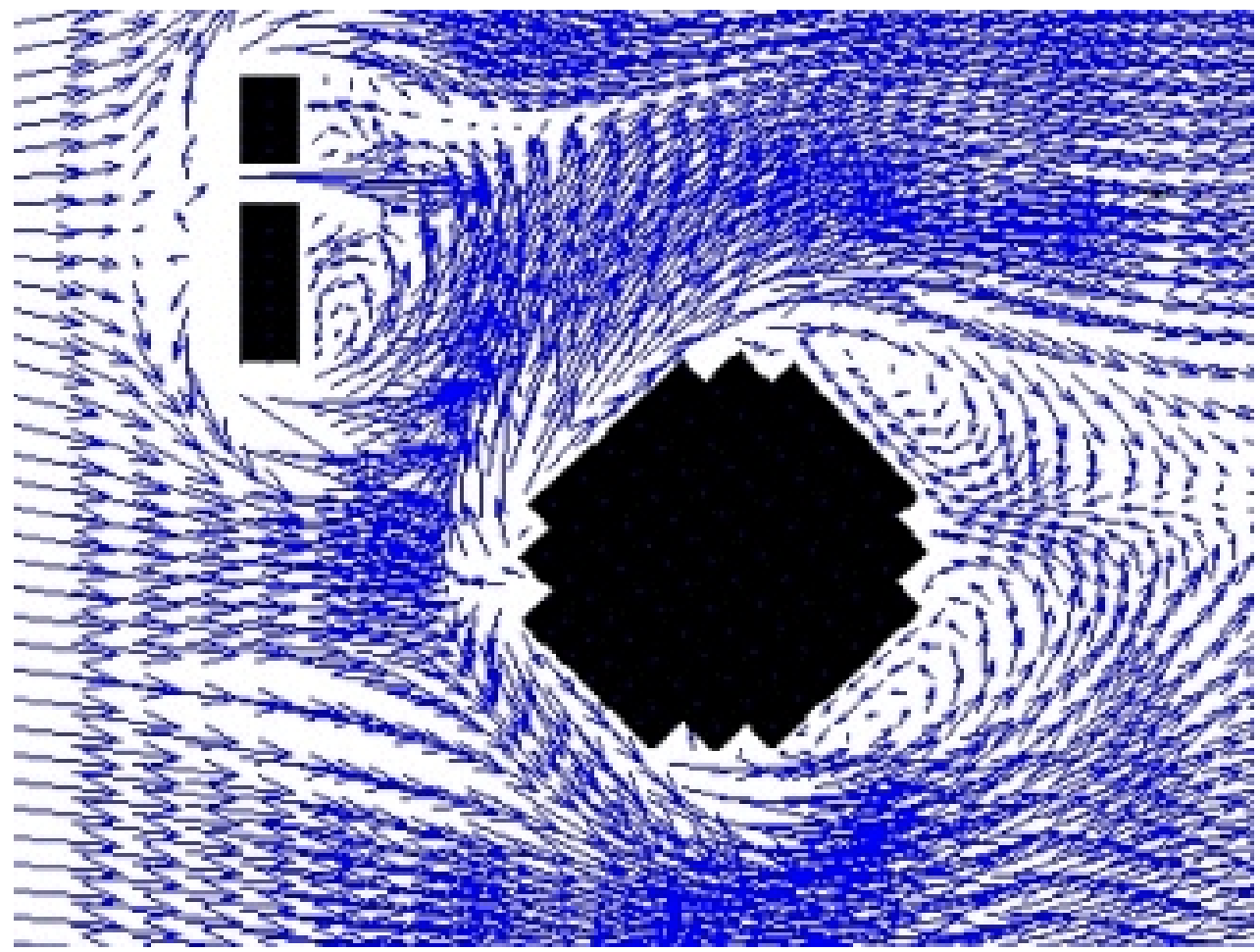

Figure 4. FEM3MP building scale simulation showing wind vectors around the Delta Center. 


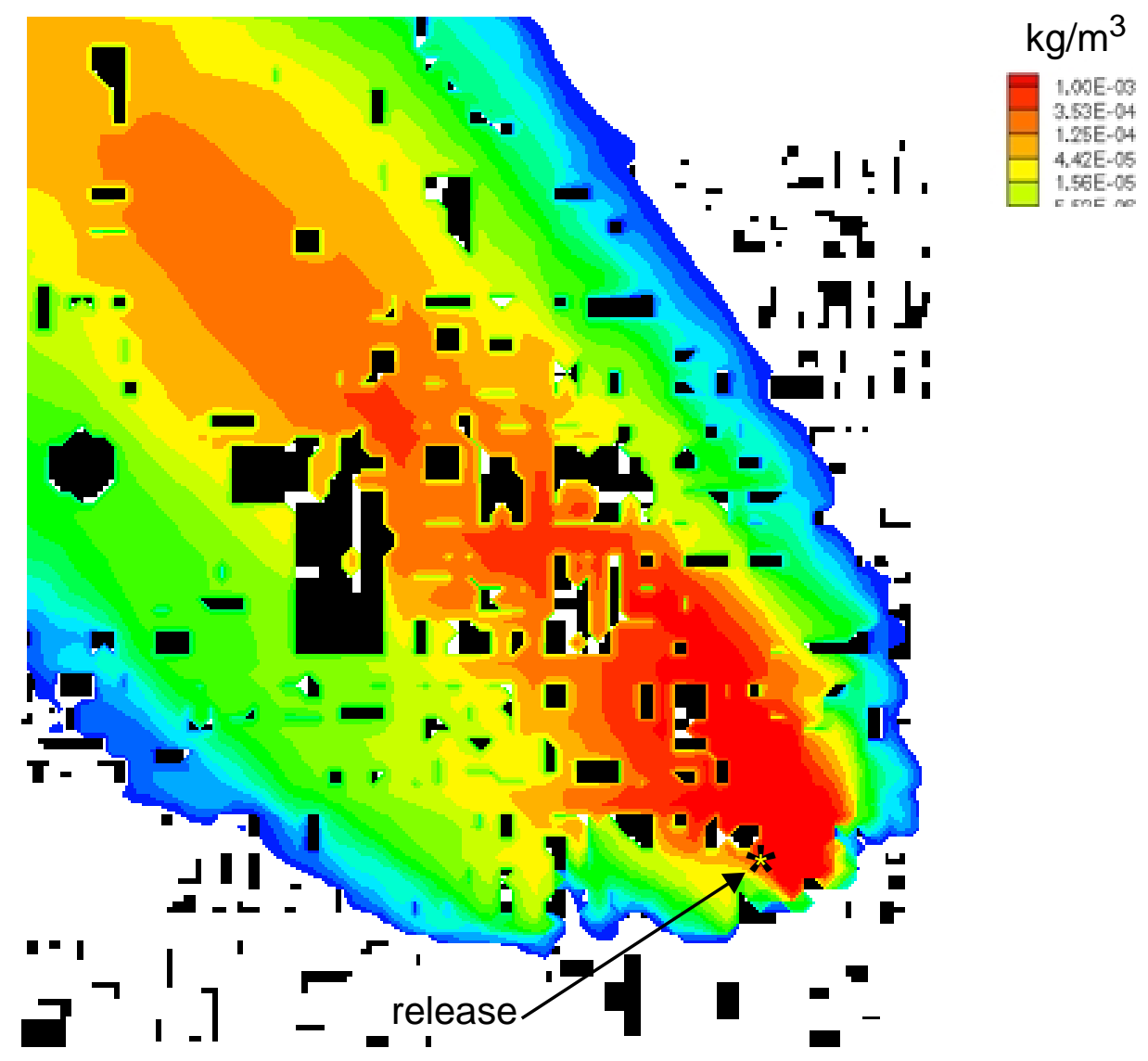

Figure 5. Tracer dispersion through downtown Salt Lake City for light southeasterly prevailing winds. Time-averaged ground-level concentrations are shown.
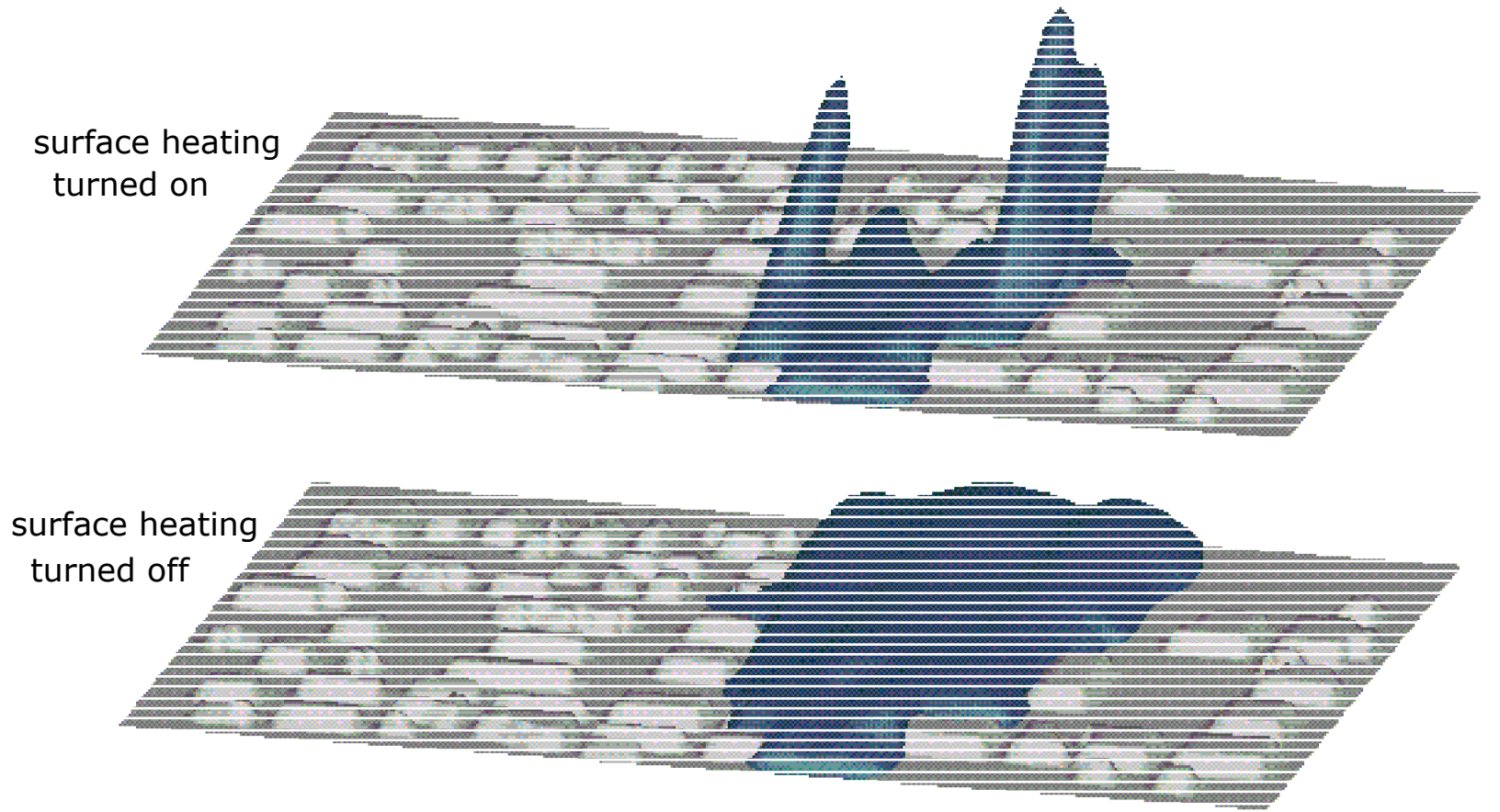

Figure 6. Example of the effect of surface heating on plume dispersion. In the upper panel, large convective eddies resulting from solar heating of the surface lift the tracer high into the air. 
$10 \times 10 \times 5 \mathrm{~m}$ resolution

$168 \times 84 \times 51=719,712$ grids

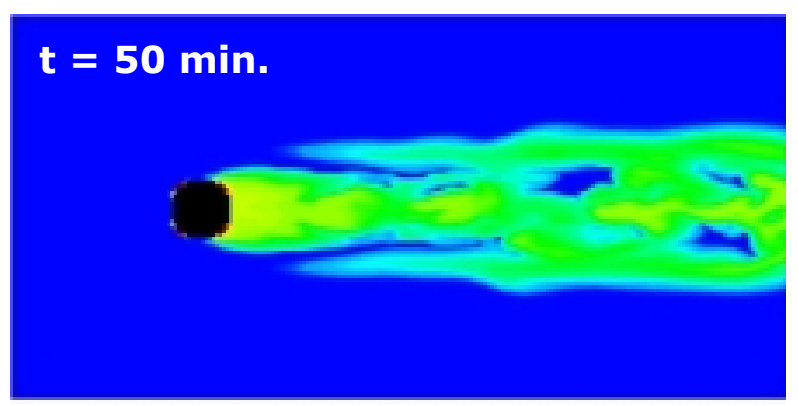

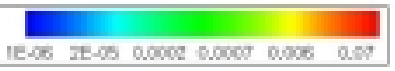

$5 \times 5 \times 2.5 \mathrm{~m}$ resolution

$336 \times 168 \times 101=5,701,248$ grids

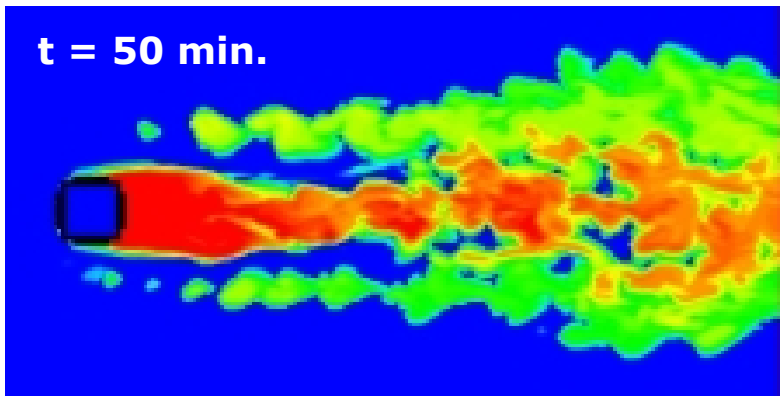

Figure 7. LES computations of tracer dispersion around a building showing the effect of doubling the grid resolution on the instantaneous concentration fields.

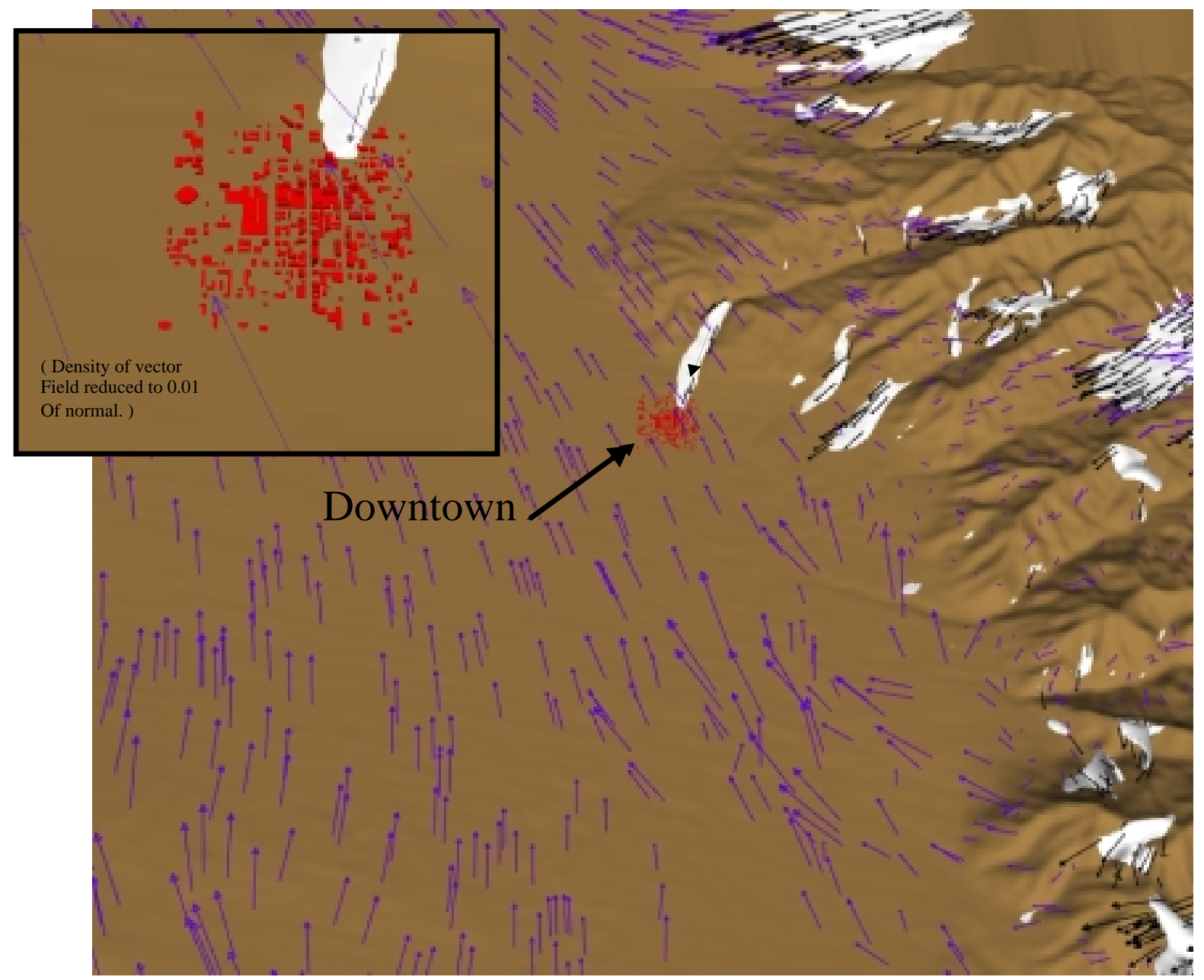

Figure 8. Salt Lake Basin early evening simulation. Purple vectors show flow at $2000 \mathrm{~m}$ above sea level ( 700m above basin floor) and black vectors flow near the surface. White isosurfaces denote areas where drainage flows undercut the upper flow. The inset zooms in on the downtown area and shows canyon drainage flows penetrating into the city. 


\section{3-d simulation}

$360 \times 128 \times 63=2,903,040$ grids

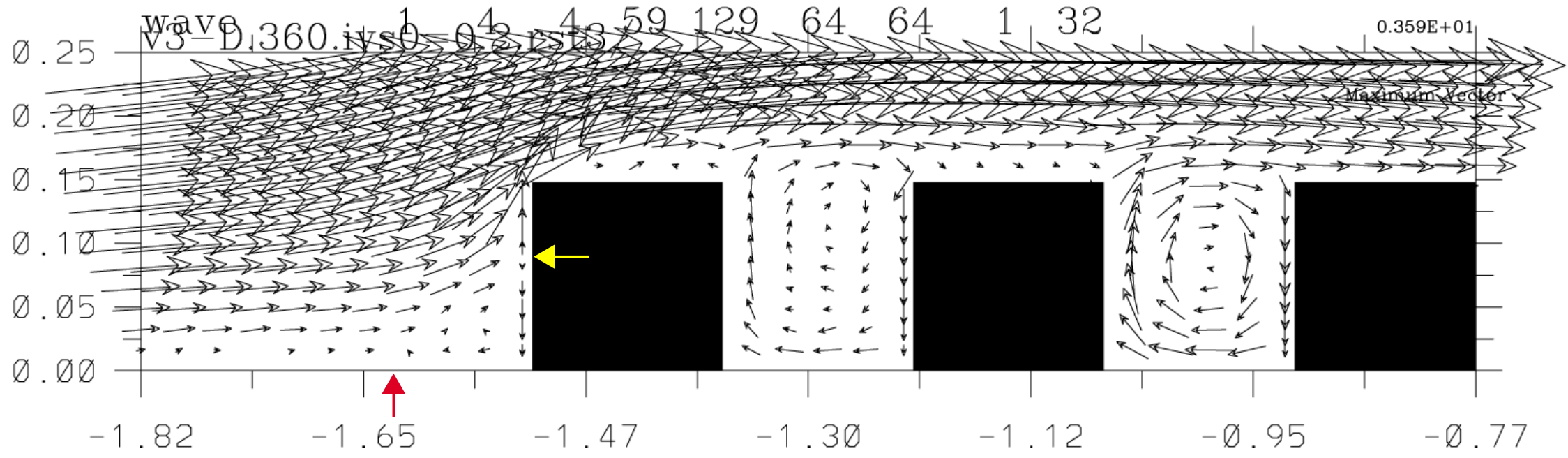

USEPA FMF Wind-Tunnel Experiments: 2-d Building Array

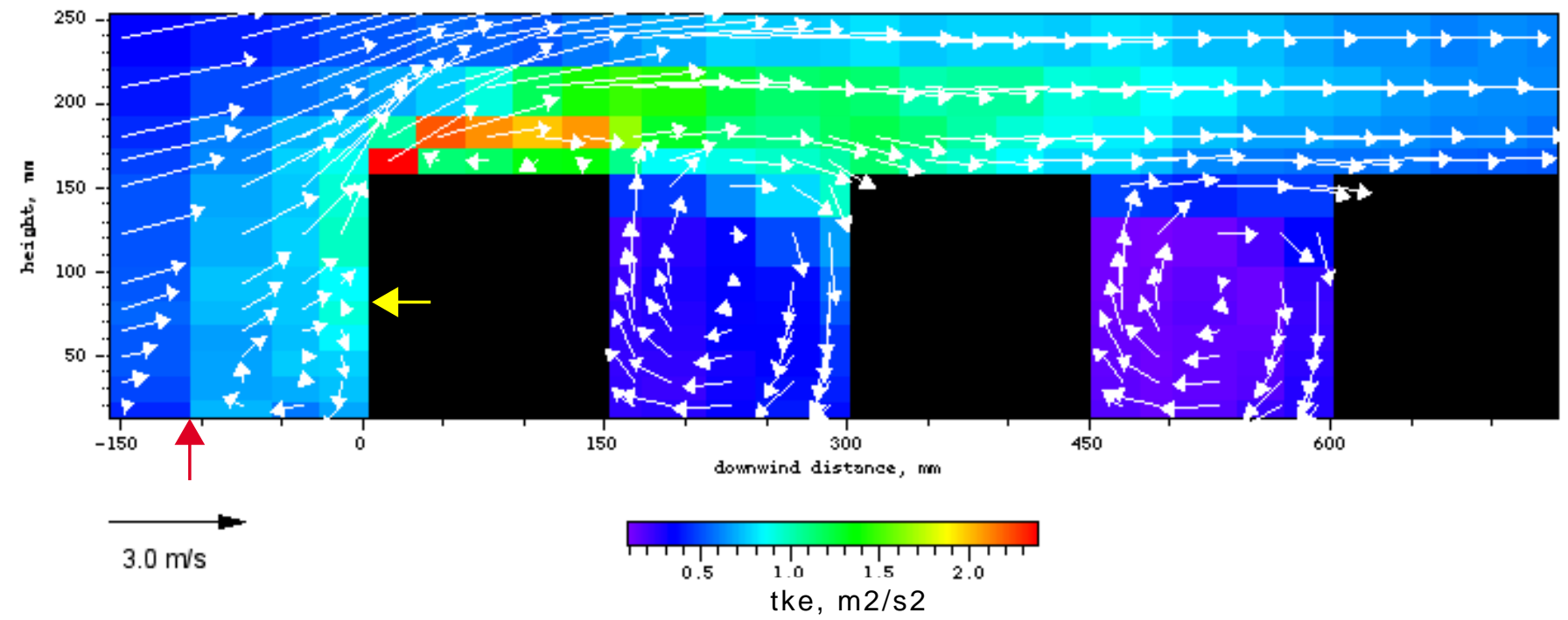

Figure 9. Comparison of the wind field around a 2-d building array computed by the model and measured in the wind-tunnel. 
Reattachment Length (Model vs. Flow Tank data)

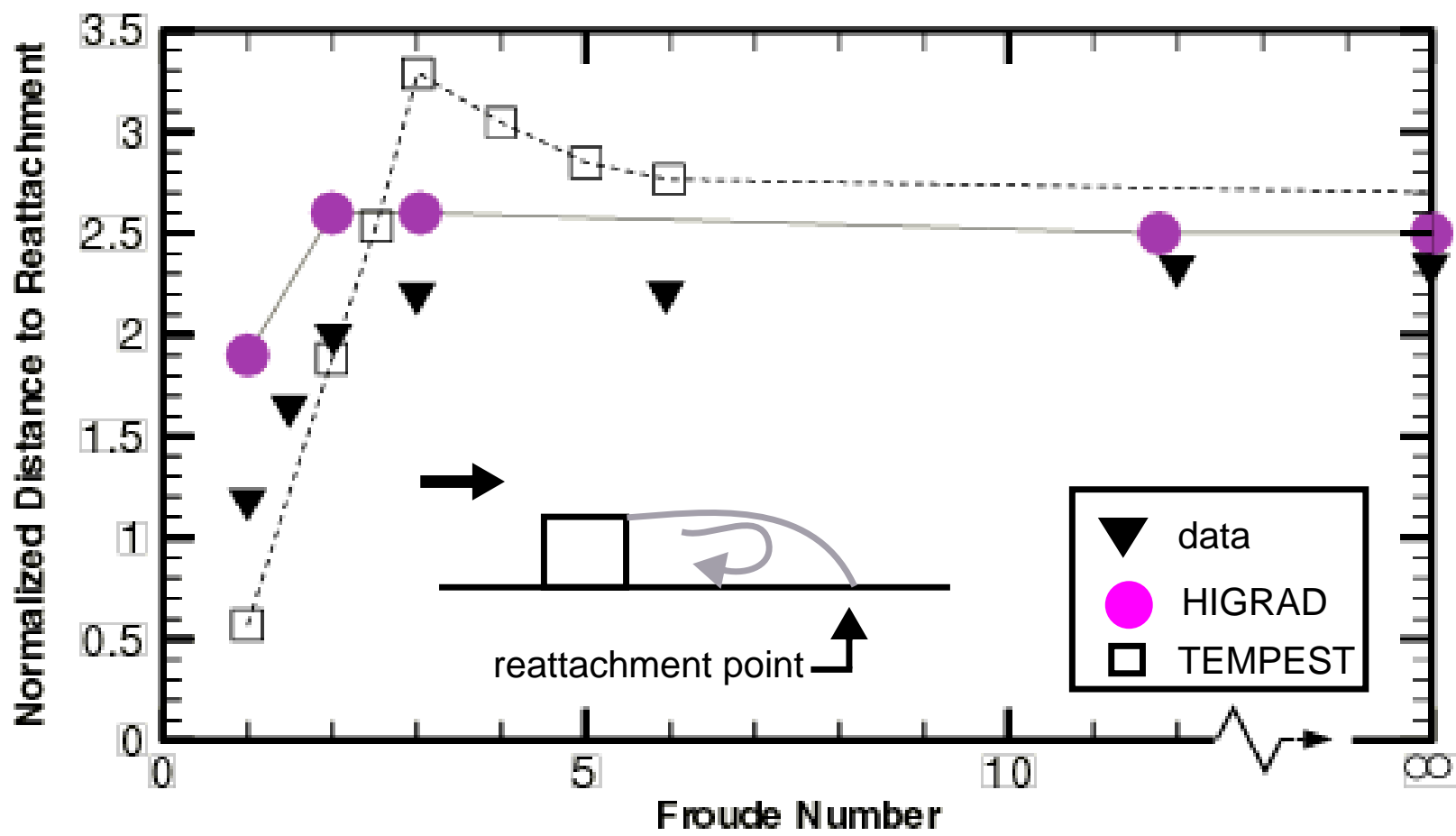

Figure 10. Comparison of reattachment length as a function of stratification. Tow tank data vs. LES model results (HIGRAD) vs. RANS model results (TEMPEST).

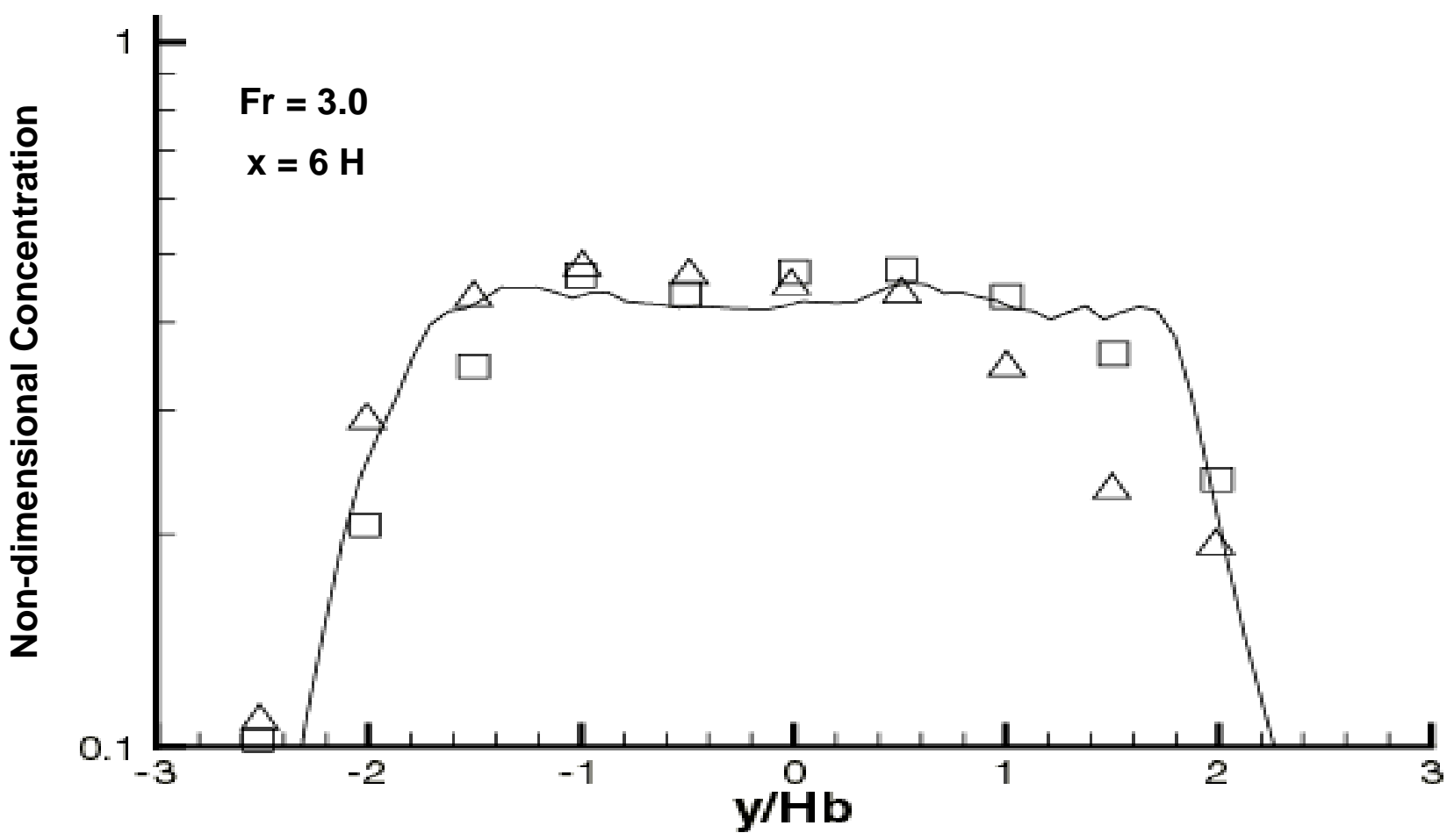

Figure 11. Lateral profile of concentration six building heights downstream of cubical building: tow tank measurements (symbols) vs. HIGRAD model results (line). 